\title{
Association of cusp energetic ions with geomagnetic storms and substorms
}

\author{
J. T. Niehof ${ }^{1, *}$, S. K. Morley ${ }^{1}$, and R. H. W. Friedel ${ }^{1}$ \\ ${ }^{1}$ Los Alamos National Laboratory, MS-D466, Los Alamos, NM 87545, USA \\ * formerly at: Boston University Center for Space Physics, 725 Commonwealth Ave, Boston, MA 02215, USA
}

Correspondence to: J. T. Niehof (jniehof@lanl.gov)

Received: 9 April 2012 - Revised: 17 September 2012 - Accepted: 29 November 2012 - Published: 6 December 2012

\begin{abstract}
Energetic ions observed in the cusp have been explained as a result of processes within the magnetosphere, but also proposed as a driver of some of those same processes. This study assesses potential connections between energetic ions observed in the cusp and geomagnetic storm and substorm activity. These connections may suggest sources of cusp energetic particles (CEPs), or imply effects of these particles on magnetospheric dynamics. We identify CEPs from six years of cusp crossings by the Polar satellite, relating them to storm and substorm onsets. CEPs showed no significant dependence on storms but did show a weak, statistically significant, increase after substorm onsets. CEPs had no significant association with subsequent storm or substorm onsets. We conclude that substorm acceleration may contribute to CEPs but CEPs are unlikely to contribute to global magnetospheric dynamics.
\end{abstract}

Keywords. Magnetospheric physics (Energetic particles, trapped; Magnetopause, cusp, and boundary layers; Storms and substorms)

\section{Introduction}

The Polar satellite (Russell, 1995) was launched on 24 February 1996 into a $1.8 R_{\mathrm{E}} \times 9 R_{\mathrm{E}}$ polar orbit, period $18 \mathrm{~h}$, inclined $>84^{\circ}$ from the ecliptic. It operated until its fuel supply was exhausted and was shut down in April of 2008. Early in the mission, Chen et al. (1997) reported observations of energetic ions in the cusp, dubbing them cusp energetic particles (CEPs). Zong et al. (2004) and Zong et al. (2005) showed observations and model field line geometries suggesting the trapping of energetic ions and electrons in the cusp.
Further studies of CEPs and their relationship to potential drivers (e.g., Zhang et al., 2005) have lead to three suggested sources, possibly acting in concert:

1. Chen et al. (1998) proposed local acceleration of particles in the cusp, particularly in diamagnetic cavities. Niehof et al. (2010) showed a long-term association between observations of CEPs and cusp diamagnetic cavities; Walsh and Fritz (2011) presented electron observations consistent with local acceleration. Other studies have examined candidate mechanisms for such acceleration. Chen and Fritz (1998) presented a correlation between local ULF wave power and energetic ions. Otto et al. (2008) modeled trapped particles in a cusp-like geometry, showing acceleration from electric fields. Others (e.g. Fritz et al., 2003; Whitaker et al., 2007) examined propagation constraints on the energetic particles, finding that particles drifting to the cusp from other candidate acceleration regions would need to violate adiabatic invariants. Nykyri et al. (2012) extended both the drift constraints and cusp modeling. However, Trattner et al. (2010) presented examples of CEPs unassociated with diamagnetic cavities.

2. At substorm onset, the stretched tail field returns to a dipole configuration. This dipolarization can accelerate particles in the tail (Birn et al., 1997). This and other acceleration mechanisms have been reviewed by Birn et al. (2012). Blake (1999) traced energetic particles in a model magnetic field and demonstrated that they could drift from the night side to high latitude on the day side, populating the cusp. Via this mechanism, Delcourt and Sauvaud (1999) showed ring current and radiation belt 
particles could access the cusp, particularly during the rapidly-varying geomagnetic field of a substorm.

Using Cluster observations of the exterior cusp, Asikainen and Mursula (2005) attributed CEPs to the day side high-latitude plasma sheet (HLPS), itself populated by energetic particles drifting from the tail. They showed a positive correlation between energetic proton fluxes in the cusp and the AE index, interpreted as a measure of substorm activity. However, AE is also a signature of magnetospheric convection, without specifically requiring substorms (e.g., Sergeev et al., 1996). The fluxes in the HLPS were also shown to correlate strongly with several other measures of magnetospheric driving. Thus although the correlation with $\mathrm{AE}$ is suggestive of a substorm source, it is an ambiguous signal.

3. The quasi-parallel bow shock is known to accelerate solar wind ions to hundreds of keV. Chang et al. (1998) argued that these ions can easily propagate to the cusp. This mechanism did not readily explain CEPs of apparent ionospheric origin; based on spectral differences between the low and high charge state CEPs, Chang et al. (2000) proposed those particles originated from magnetospheric leakage. Trattner et al. (2001) similarly concluded that high charge state CEPs below $150 \mathrm{keV}$ were accelerated at the shock, and higher energy CEPs were accelerated inside the magnetosphere before leaking to the cusp.

The origin of CEPs would be particularly important if they influence the global magnetospheric system. Fritz et al. (2000) proposed that CEPs drift to the night side and form a component of the ring current, partial ring current, and radiation belts. In this hypothesis, CEP events should be followed by signs of energetic particle activity elsewhere in the magnetosphere; whereas a magnetospheric leakage source for CEPs implies CEP observations should follow magnetospheric activity. This study examines associations between CEP events and geomagnetic activity in either direction, testing both the hypothesis of geoeffective CEPs and the leakage theory of CEP origin.

This paper first presents the data sets used in our study, including event selection criteria. Section 3 describes the key technique of association analysis, which we apply to these sets in Sect. 4. Finally we discuss the implications of this analysis for the role of CEPs in the magnetosphere.

\section{Data sets}

Cusp passes and CEP events are based on the events from Niehof et al. (2010), derived from the Polar satellite observations. Their derivation is briefly summarized here. The Polar satellite's eccentric orbit, with apogee at high latitude, provided excellent coverage of the cusp. Cusp signatures were sought in the region where the Polar satellite was on the day side $\left(X_{\mathrm{SM}}>0\right)$, at geocentric distance $>6 R_{\mathrm{E}}$, and magnetic latitude $>=25^{\circ}$. Within this region, moments of the proton distribution from the HYDRA hot plasma analyzer (Scudder et al., 1995) were monitored for high density (fiveminute running average $>0.5 / \mathrm{cc})$ and stagnant flow $\left(X_{\mathrm{GSM}}\right.$ speed $<100 \mathrm{~km} \mathrm{~s}^{-1}$ ). Such regions were labeled part of the cusp. Energetic particles within the cusp were observed by the CAMMICE MICS instrument (Fritz et al., 2004). CEPs were identified from the definition of Chen and Fritz (2005): a MICS $>40 \mathrm{keV}$ ion count rate exceeding the instrument background by $3 \sigma$. Applying these criteria to the six years when MICS was in operation (April 1996 through March 2002), we found 1126 passes through the cusp, 915 of which had energetic particle signatures.

For determining the timing of geomagnetic storms, we used a high-resolution rederivation of the Dst index (Gannon and Love, 2011). This index was pressure corrected with the model of O'Brien and McPherron (2000). From this Dst*, storm onset times were identified by eye. We searched the data from April 1996 through March 2002 for classic storm signatures (e.g. Loewe and Prölss, 1997) where Dst* reached at least $-30 \mathrm{nT}$. Two of us (SKM and JTN) independently determined onset times from this set of 170 candidates, looking for an abrupt change to a strongly negative slope in Dst* from equilibrium values near zero. We then reviewed these independent onset determinations and discarded 14 events which showed activity in Dst*, but where we could not identify a clear storm signature. Of the remaining 156 candidates, the median difference between our onset determinations was twelve minutes, demonstrating both a consistency of selection and the benefit of an index with finer resolution than the standard one-hour Kyoto Dst. On a final pass through the remaining events, we readily identified one or the other of our onset selections as better representing onset, and used this "consensus" onset list for our analysis. Figure 1 shows a superposed epoch plot of these 156 events, with a clear onset signature, giving confidence in our selection of storm onsets. (The minimum is somewhat obscured by storm-tostorm variability in the length of the main phase; the plot is not normalized.)

Beginning at these onset times, we identified storm minima times algorithmically. Each minimum was selected from a period starting at onset and lasting:

\section{At least $3 \mathrm{~h}$ but not more than 30}

2. At least until Dst* passed below $-30 \mathrm{nT}$

3. Subject to the above constraints, until Dst* recovered to $50 \%$ of its minimum value.

We used two existing substorm lists and generated one new one for this study. For the new list, we sought a global substorm signature that could be directly tied to energetic particle acceleration. Birn et al. (1997) attributed substorm energization to the dipolarization of the magnetotail. This 


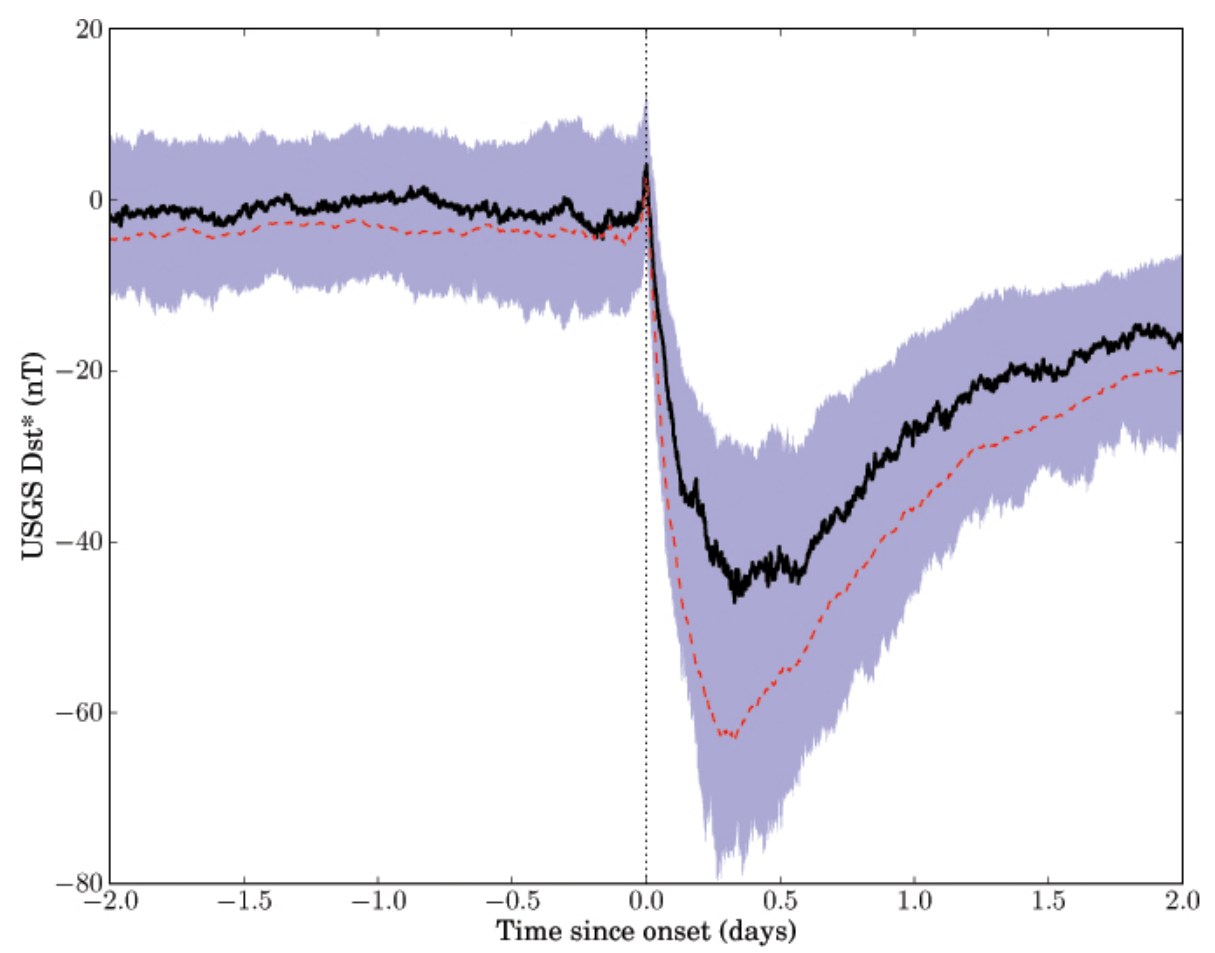

Fig. 1. Superposed epoch plot of Dst* as a function of time since storm onsets identified as described in the text. Plotted here are the superposed epoch median (black line), mean (dashed red line), and interquartile range (blue shaded region).

dipolarization should provide a clear signature of increased energetic particle availability in the magnetosphere. According to the leakage hypothesis, then, dipolarization events and CEP observations should be associated.

The technique of Liou et al. (2002) was applied to identify dipolarizations from Geostationary Operational Environmental Satellites (GOES) magnetic field data. The magnetic field vectors $\boldsymbol{B}$ were converted to the VDH coordinate system: $H$ antiparallel to the Earth's magnetic dipole, $V$ in the plane of the magnetic equator and radially outward, and $D$ completing the right-hand triplet. Then the magnetic elevation angle is

$\tan ^{-1}\left(-\frac{B_{H}}{B_{V}}\right)$

A dipolarization event was defined as an increase in elevation angle of $10^{\circ}$ in $10 \mathrm{~min}$ or less. No minimum time between dipolarizations was required; however, a single dipolarization event was assumed to last as long as the elevation angle maintained this slope. The resulting list showed 4265 dipolarizations for the study period April 1996 through March 2002.

Cai et al. (2006) found that dipolarization signatures were clearly visible from 20:00-02:00 magnetic local time (MLT), with onset between 22:00 and 00:00. Thus this substorm set was limited to times when at least one GOES satellite was positioned at MLT 20:00-02:00. Under normal operation, two satellites were available, separated by $60^{\circ}$ and providing ten hours of coverage each day. Liou et al. (2002) inferred expansion of the dipolarization region of $4.9^{\circ} \mathrm{min}^{-1}$ westward and $6.1^{\circ} \mathrm{min}^{-1}$ eastward, resulting in a worst-case $12 \mathrm{~min}$ delay between onset (at 00:00 MLT) and measurement by a satellite (at 20:00 MLT).

Energetic ions injected in substorms would take time to drift to the day side; the gradient curvature drift time for equatorially-mirroring particles in a dipole field gives an estimate (e.g. Hess, 1968, p. 34):

$\tau_{D} \sim \frac{44}{L E}$

where $\tau_{D}$ is the orbit period (in minutes), $L$ the L-shell, and $E$ the particle energy in $\mathrm{MeV}$. At geosynchronous orbit $\left(6.6 R_{\mathrm{E}}\right)$, a $40 \mathrm{keV}$ proton (the minimum energy for the CEP definition of Chen and Fritz, 2005) has a drift period of approximately $2.8 \mathrm{~h}$; a $200 \mathrm{keV}$ ion (near the upper end of the MICS range), $0.56 \mathrm{~h}$. Reeves et al. (1991) presented measurements of drift periods for electrons at geosynchronous orbit based on drift echos from substorm injections. These periods ranged from nearly $4 \mathrm{~h}$ at $37 \mathrm{keV}$ to $0.6 \mathrm{~h}$ at $245 \mathrm{keV}$. They note consistency with the dipole approximation, explained by the expected relaxed state of the field line post-substorm. As gradient-curvature drift speeds depend on particle energy per charge and pitch angle, but not mass or the sign of charge, these results are applicable to ions as well.

Half an orbit would bring an ion to the dayside, so we estimate $15-120 \mathrm{~min}$ between a substorm and possible CEP 
observations potentially caused by that substorm. Thus, even the fastest energetic particles could not arrive at the dayside from the tail before the dipolarization front reached GOES. We also expect the arrival of energetic ions in the cusp to be dispersed over about 100 min between the arrival of the most energetic ions and the least energetic.

We complemented this substorm list with two lists from the literature. Frey et al. (2004) identified 2437 substorms from two-minute cadence IMAGE-FUV images taken between 16 May 2000 and 31 December 2002 (henceforth the "Frey list."). They required a brightening of the aurora, expansion to the poleward boundary of the auroral oval, and an expansion in local time. Liou (2010) applied similar criteria to the Polar satellite UVI data ( 37 s cadence), finding 2003 substorms from 30 March 1996 through 5 February 2000 (the "Liou list.") Combining these lists, as suggested by Liou (2010), yields 3874 substorms during this study. This period does not have continuous coverage, as the orbits of the Polar satellite and IMAGE did not permit continuous auroral observations. The Liou list contains the time of both the last image before the auroral brightening was observed and the first showing the brightening. For consistency with the Frey list, the second time was chosen as the substorm onset time. Boakes et al. (2009) noted that the Frey list may include nonsubstorm events, as they do not all show energetic particle injections at geosynchronous orbit. The Liou list may require a similar caution.

Section 4.2 compares the Liou-Frey list with that derived from GOES-measured dipolarizations.

\section{Technique}

The data sets in Sect. 2 provide lists of times when the Polar satellite was in the cusp, the subset of those times with energetic particles, and onset times of storms or substorms. We seek any significant association between the events in the first class (CEP events) and those in the second (storms, substorms). Niehof and Morley (2012) provide an extensive examination of techniques for determining connections between two different series of discrete events (point processes) (Cox, 1955) and the significance of the connection; here we summarize the theory. Previous applications of association analysis to space science include Hsu and McPherron (2002) and Morley and Freeman (2007).

Consider two time series of different types of events, here called series A and series B. Each series is completely described by a list of times when events of the appropriate type occurred. In this study, series A is a set of storm or substorm times, and series B contains times when the Polar satellite observed CEPs, at a one-minute resolution.

Given measurements of a series of $N_{\mathrm{A}}$ type A events, the $i$-th event is denoted $a_{i}$. The $j$-th event of $N_{\mathrm{B}}$ type B events, is similiarly denoted $b_{j}$. Assume a time lag $u$ between the series of events, and a half-window $h$ representing an uncer- tainty in the timing of events or in the delay between them. The $i$-th individual association on series A is defined (Morley and Freeman, 2007):

$c_{\mathrm{A}, i}=\#\left\{\left|b_{j}+u-a_{i}\right|<h\right\}$

where $\#\{\}$ is the number of elements in set \{\} . In the zero-lag case, this is the number of $\mathrm{B}$ events $b_{j}$ which fall within halfwindow $h$ of a particular A event $a_{i}$. A non-zero lag shifts series B in time with respect to series A.

The association number as a function of lag and halfwindow is the sum of the individual associations:

$n(u, h)=\sum_{i=1}^{N} c_{\mathrm{A}, i}$.

This can be described as the number of occurrences where an A-type event and a B-type event are within a half-window of each other. It is independent of the choice of which series is assigned to series A and which to B. It is also a different from a conditional probability: the association number does not presuppose the occurence of one type of event.

With no a priori knowledge of the lag between events, the association number is calculated by Eq. (3) for a large number of lags $u$ covering the expected range of lags between the two sets of events. Here we use lags of -5 to 5 days, in five minute increments. The expected drift periods (Sect. 2) span a range of approximately $100 \mathrm{~min}$, largely captured with a half-window of $30 \mathrm{~min}$. Prior knowledge of the drift period itself is not required for the association analysis.

At very high lags, we expect any physical association between the series to be broken. Thus the association number at these high lags should tend to that of chance association (Brillinger, 1976). This is called the asymptotic association number, $n(u \rightarrow \infty, h)$. Association numbers above the asymptotic association number indicate an association greater than chance. The association analysis says nothing about causality, only the temporal order of the association.

To determine the significance of the association, we construct confidence intervals on the association number using a bootstrap technique (Efron, 1979). For each lag $u$, the set of $N_{\mathrm{A}}$ individual associations $c_{\mathrm{A}, i}$ are resampled with replacement to construct a surrogate set, also of length $N_{\mathrm{A}}$ (cf. Morley and Freeman, 2007). The individual associations in this surrogate set are summed, yielding a surrogate association number. Repeating this process several thousand times results in a set of surrogate association numbers which reflect the statistics of the population, and a $95 \%$ confidence interval (c.i.) can be chosen from the 2.5-th and 97.5-th percentile of the surrogates. If the asymptotic association number is outside this c.i., the null hypothesis of chance association can be rejected at the $95 \%$ level. In this study, we constructed confidence intervals from 10000 surrogates at each lag.

The size, shape, and location of the cusp changes in response to geomagnetic activity and external drivers of that activity. Any association with CEPs must be distinguished 
from an association with conditions that make it easier for the Polar satellite to observe the cusp, such as an increase in solar wind pressure. The $95 \%$ c.i. for the association number for cusp events with storms or substorms is compared to the association number of CEP events with the same. There are, by definition, fewer CEP events than cusp events. To allow a meaningful comparison, the asymptotic association number for each association was calculated from the median of association numbers for absolute lags of greater than three days, which should be well beyond any systematic association. The association number for CEP events was scaled so that the asymptotic association numbers match those for the cusp; the confidence intervals were scaled by the same ratio. The resulting CEP numbers were then divided by the cusp association number, removing variability of the cusp association with lag. This eliminated periodicities in the association due to limited sampling in both the CEP and substorm data sets and highlighted differences between CEP and cusp observations. The relative association number for cusp events was thus fixed at 1.0. The c.i. around this value varies with lag and can be compared to the c.i.'s for the CEP relative association number. Where the lower bound of the relative CEP c.i. exceeded the upper bound of the cusp c.i., we can be $95 \%$ confident $\left(.975^{2}\right)$ that CEPs are more strongly associated with storms or substorms than the cusp is.

The bootstrap resampling requires independence of the individual associations $c_{\mathrm{A}, i}$ (not individual events $a_{i}$ ). In practice this condition can be met provided that events $a_{i}$ are sufficiently spaced such that a B-type event $b_{j}$ which falls within a half-window of $a_{i}$ is outside the half-window of preceding or following events, $a_{i-1}$ and $a_{i+1}$ (see also Niehof and Morley, 2012). This study's thirty minute half-window is much smaller than the time between storm onsets (a minimum of one day, eight hours in this study's list) or the hours between substorms (Borovsky et al., 1993; Freeman and Morley, 2004), our choices for series A.

The association analysis, related bootstrapping, and plotting were performed with the PoPPy module of the SpacePy package (Morley et al., 2010).

\section{Results}

\subsection{CEPs and storms}

Geomagnetic storms are identified by disturbances in the Dst index, usually interpreted as an intensification of the ring current (e.g. Loewe and Prölss, 1997). If the hypothesis of Fritz et al. (2000) is correct and CEPs contribute significantly to the ring current, we might expect some association between CEP observations and the most dramatic variation in the ring current. We calculated the association between storm onsets and CEP observations by the Polar satellite over lags ranging from -5 to 5 days at 5 min resolution.
The association between storm onsets and CEP observations by the Polar satellite (Fig. 2), as compared to the association with CUSP observations, is well within that explained by chance. The confidence interval for the storm-CEP association largely overlaps that for the storm-cusp association. Indeed, the association number at asymptotic lags is very similar to that across the entire range of lags, and no peaks are apparent of even low significance.

Although the presented association plots show lags from -70 to $70 \mathrm{~h}$ ( $\sim 3$ days), the association analysis was performed for -5 to 5 days to determine the asymptotic association number, and a full bootstrap calculation was performed for -4 to 4 days to search for any features of interest at large absolute lags. None were found. Thus we can be confident that the presented asymptotic association number indeed represents the level of chance association, unless CEPs and storm onsets are associated, to an identical level, at both very highly positive lags (CEPs preceding substorms) and highly negative lags (CEPs following substorms).

The association analysis was repeated using the storm minima as the event time rather than the onsets (not shown), with similar results and no significant peaks. Thus storm onsets and minima occur independently of CEP observations. Given this independence, there is no indication that CEPs trigger storms. Similarly, dramatic stormtime enhancements in the ring current (median of $-45 \mathrm{nT}$ in this study) in less than ten hours show no association with CEPs over the following several dozen hours. Were CEPs continually fed by the ring current, and were this a significant source, we would expect some response to such a strong enhancement. This does not, however, eliminate the ring current as a potential CEP source, with energetic ions only leaking to the cusp under particular conditions, such as substorms (Sect. 4.2).

\subsection{CEPs and substorms}

Figure 3 shows the association with subtorms for CEPs and cusp observations, based on the combined Frey and Liou lists. The largest peak of CEPs relative to cusp occurs for lag of $-595 \mathrm{~min}$, i.e. the CEP observations lead the substorms by $10 \mathrm{~h}$. This peak is, however, significant at only the $89 \%$ level; chance association would find peaks this significant for about 327 of the 2881 lags in the analysis. Indeed, there is a $91 \%$ significant depression at $109 \mathrm{~h}$ (4.54 days, not shown), well into the asymptotic region.

The Liou list is based on data from the Polar satellite, which was also used for CEP and cusp observations. Correlations between the auroral observations and cusp passes may have caused artifacts in Fig. 3; therefore, Fig. 4 shows the same analysis using only events from the Frey list. Periodicities in the cusp confidence interval disappear. However, there is still no significant association. These data provide no support for any association between CEPs and substorms, in either direction. 


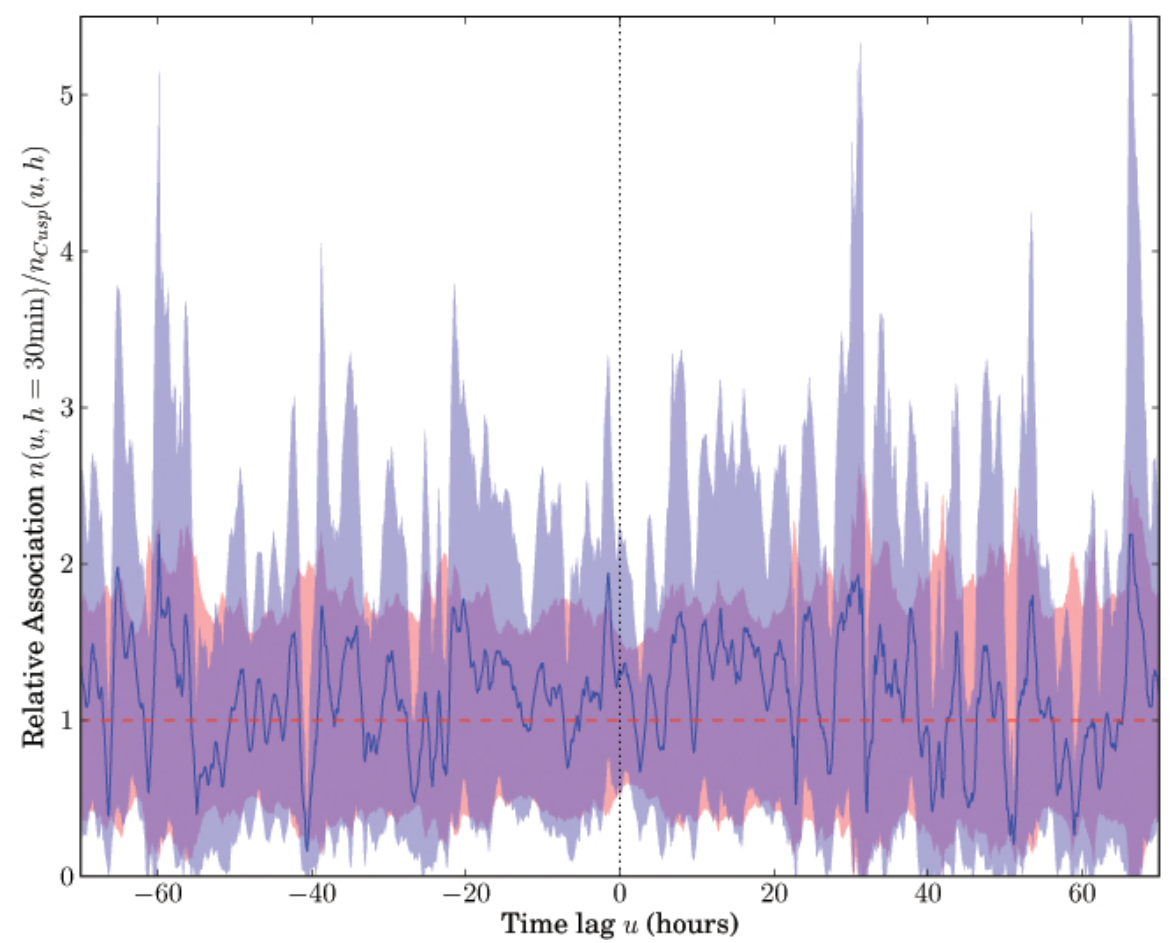

Fig. 2. Association of CEPs (blue) and cusp (red) with storm onsets identified from Dst*. Normalized to match asymptotic association numbers and so cusp association is 1.0. Lines represent the calculated association number; shaded areas indicate $95 \%$ confidence intervals; overlapping c.i. are magenta.

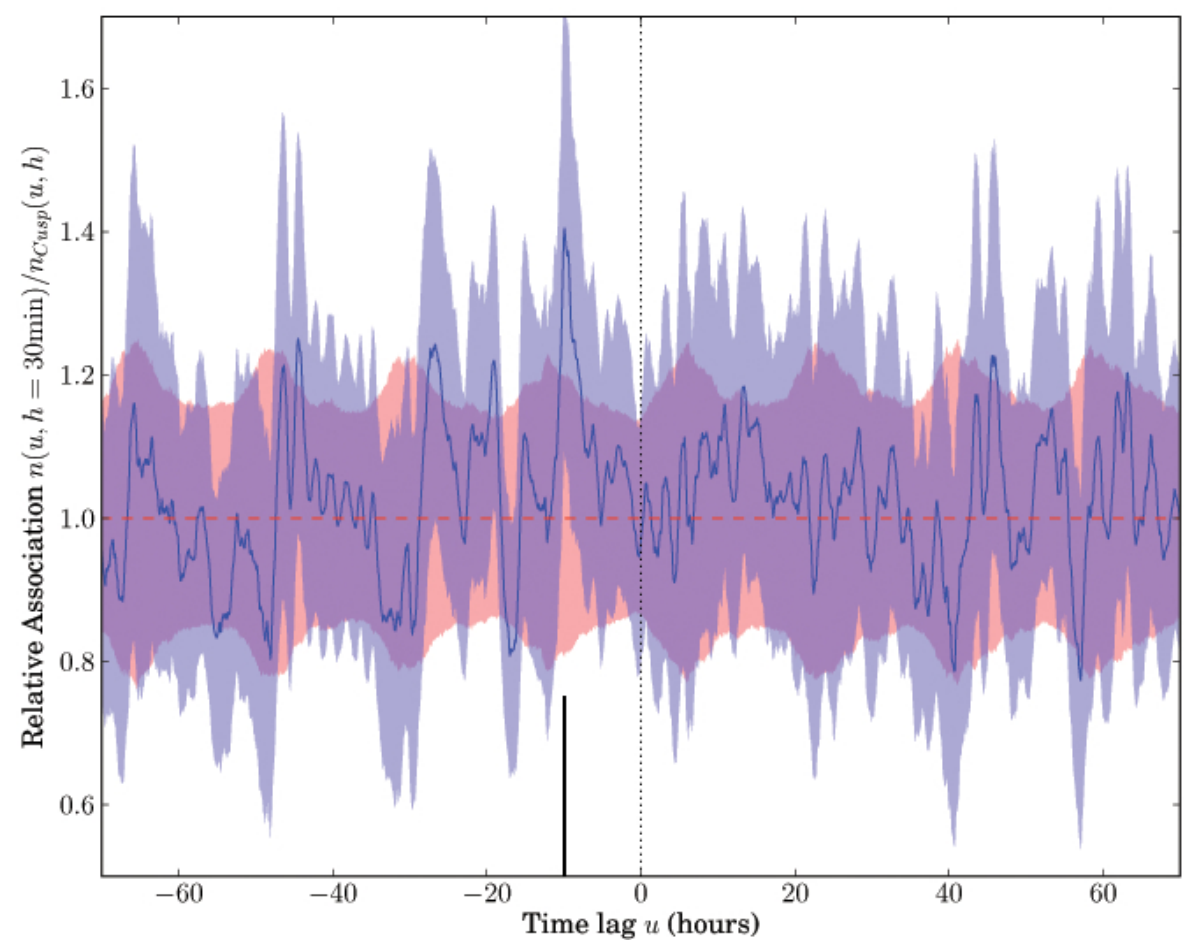

Fig. 3. Association of CEPs (blue) and cusp (red) with substorms from the Frey-Liou list. Normalized to match asymptotic association numbers and so cusp association is 1.0. Lines represent the calculated association number; shaded areas indicate $95 \%$ confidence intervals; overlapping c.i. are magenta. Heavy black lines mark features discussed in text. 


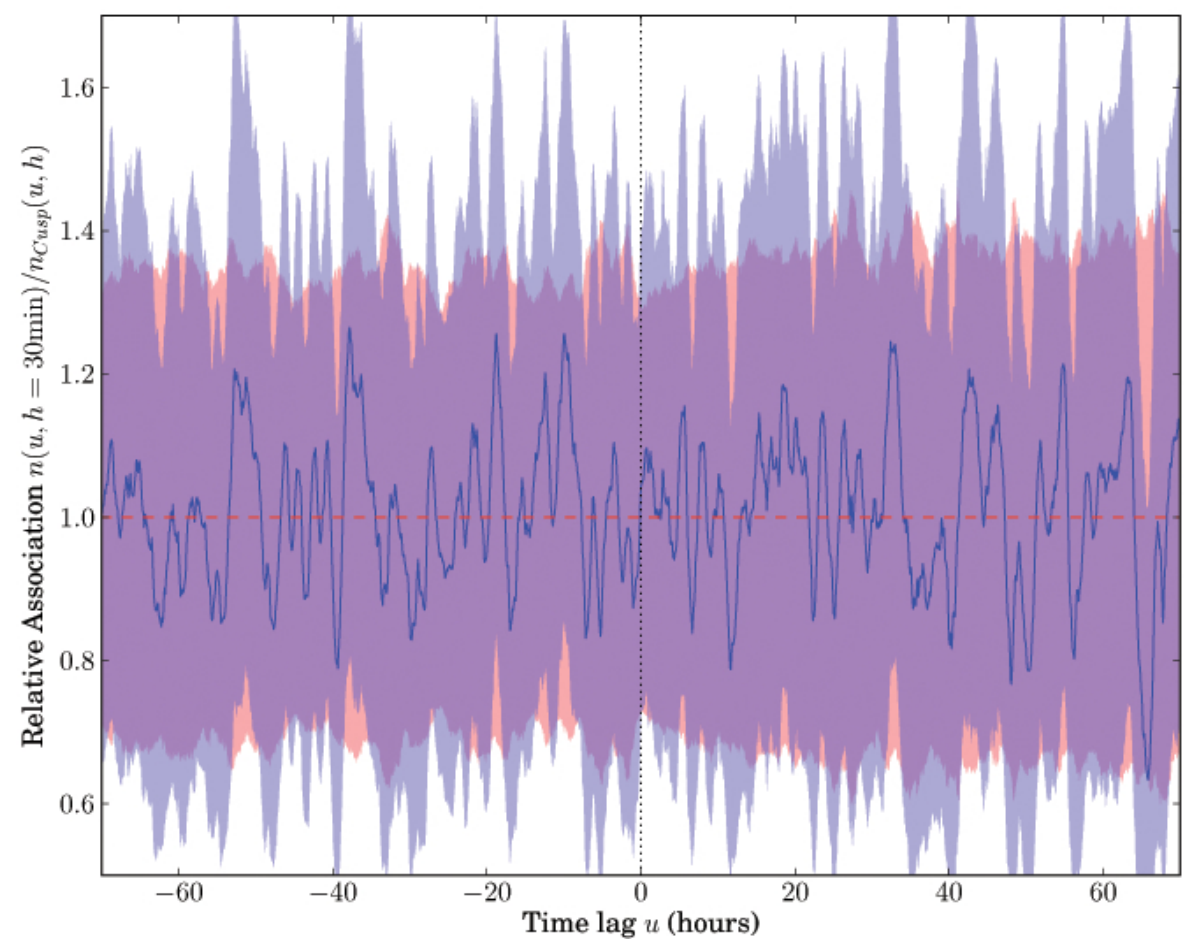

Fig. 4. Association of CEPs (blue) and cusp (red) with substorms from the Frey list only. Normalized to match asymptotic association numbers and so cusp association is 1.0. Lines represent the calculated association number; shaded areas indicate $95 \%$ confidence intervals; overlapping c.i. are magenta.

The same analysis for the GOES dipolarization-based substorm list is in Fig. 5. Here, the CEP association with substorms is greater than the cusp association at about the $95 \%$ level for lags from approximately 3 through $3.5 \mathrm{~h}$, peaking at $96 \%$ significance. This suggests some direct and immediate contribution to CEP occurrence from ions accelerated in substorms, with a drift period to the dayside consistent with the drift periods presented in Sect. 2. The increased association is apparent, although below the $95 \%$ level, for nearly $20 \mathrm{~h}$. This may be partially an artifact of the Polar orbit: since the Polar satellite sampled the high-altitude dayside once per $18 \mathrm{~h}$ orbit, for some events it could be several hours between the appearance of CEPs and their observation. A similar heightened association, around $-7 \mathrm{~h}$, is short and below the $95 \%$ confidence limit. It may well represent random variability (as discussed above).

Indirect mechanisms, such as trapping in the HLPS (Asikainen and Mursula, 2005), are not excluded by this analysis; neither are non-substorm mechanisms. For any proposed CEP source, events can be identified which appear consistent with only that source (e.g., Whitaker et al., 2007). However, this study demonstrates support for a specific variation on the magnetospheric leakage origin of CEPs suggested by Blake (1999) and others, one significant enough to appear in a long-term statistical study rather than case studies.
Neither list yielded a statistically significant association between substorms and CEPs at negative lags, i.e. for CEPs occurring before substorms. This association would be expected if CEPs were to somehow contribute to triggering substorms. Its lack implies CEPs are not directly involved in substorm processes.

The Frey and Liou events are not necessarily accompanied by energetic particles at geosynchronous orbit. Using a small (five month) subset of the Frey list, Boakes et al. (2011) found only $33 \%$ of the listed substorms were associated with classic energetic particle injection signatures at geosynchronous, $37 \%$ showed some other energetic particle change, and $30 \%$ showed no geosynchronous energetic particle fluctuations at all. The list may contain a substantial number of injectionless events, or events with injections too weak to reach geosynchronous orbit, weakening any association with energetic ions. A clear association, however, does not require a one-to-one correspondence between two series: Morley and Freeman (2007) found a significant association between substorms and triggers in general, but demonstrated that only $25 \%$ of substorms showed a one-to-one correspondence.

The combined Frey-Liou lists contained 3874 events in the study period; of these, 1326 had GOES coverage for the thirty minutes before and after the onset. Of these events, 965 showed dipolarizations at GOES. When compared to the 


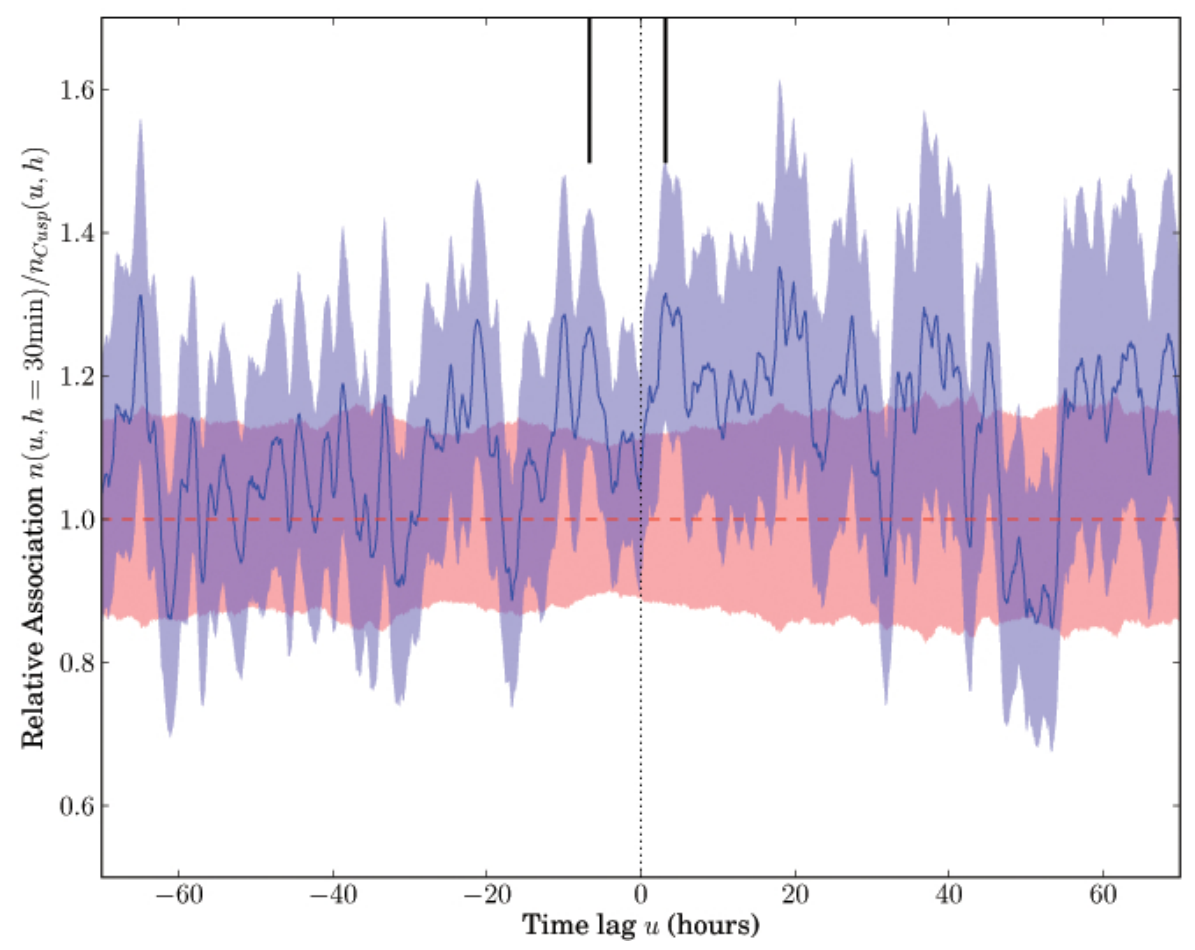

Fig. 5. Association of CEPs (blue) and cusp (red) with substorms detected by GOES dipolarizations. Normalized to match asymptotic association numbers and so cusp association is 1.0. Lines represent the calculated association number; shaded areas indicate $95 \%$ confidence intervals; overlapping c.i. are magenta. Heavy black lines mark features discussed in text.

4265 total dipolarizations measured by GOES, it is clear that these lists have substantial overlap but many events unique to each.

Ions gradient-curvature drift to the west in the geomagnetic field, so energetic ions drifting from the tail to the day side would be expected to appear first in the dusk sector. Figure 6 shows the MLT distribution of energetic ions in the cusp. MLT has been corrected for aberration due to nonradial solar wind flow and the Earth's orbital motion, after the method of Dmitriev et al. (2003): the coordinate system for determining MLT was created by rotating the SM system about the z-axis by angle $\delta_{Y}$ :

$\delta_{Y}=\tan ^{-1}\left(\frac{V_{Y}+30 \mathrm{~km} \mathrm{~s}^{-1}}{\left|V_{X}\right|}\right)$

where $V_{Y}, V_{X}$ are solar wind velocity components from the five minute resolution OMNI dataset ${ }^{1}$ (Papitashvili and King, 2006). This rotation places MLT 12:00 at the point facing directly into the upstream flow, rather than at the subsolar point. Figure 6 only includes data with four continuous hours of preceding GOES coverage at MLT 22:00-02:00. The hashed bars include data with no dipolarization during these four hours; solid gray bars include data with at least one dipolarization. Error bars show the $1 \sigma$ range from binomial statistics:

\footnotetext{
${ }^{1}$ Available at http://omniweb.gsfc.nasa.gov/.
}

$\sigma=\sqrt{\frac{p(1-p)}{N}}$

where, for each MLT bin, $p$ is the fraction of time in the cusp with CEPs present and $N$ is the number of samples in the cusp. $N$ is calculated assuming five minutes of observation constitute one independent measurement, i.e, for a bin with $t$ minutes of cusp observations, $N=t / 5 \mathrm{~min}$.

Changes in the MLT distribution post-dipolarization are quite apparent. To evaluate their significance, an expected distribution was constructed. Starting with the nodipolarization distribution, the fraction of cusp observations with energetic particles for each bin was scaled so that the total such fraction across all bins (weighted by the number of measurements in each bin) matched the measured fraction post-dipolarization. Comparing the actual distribution post-dipolarization with the expected yielded $\chi^{2}=28.3$, or a $3 \times 10^{-6}$ probability of the distributions being the same.

Although this change in distribution was significant, it was not limited to the expected dusk-side enhancement: CEP observations expanded into the morning sector as well. It is possible that the cusp/high-latitude trapping region could no longer trap ions in the reconfiguring magnetic field of a substorm, or that the additional ions observed were transiting the cusp without being confined there. Also, ions trapped in the cusp will orbit the cusp funnel, alternating eastward and 


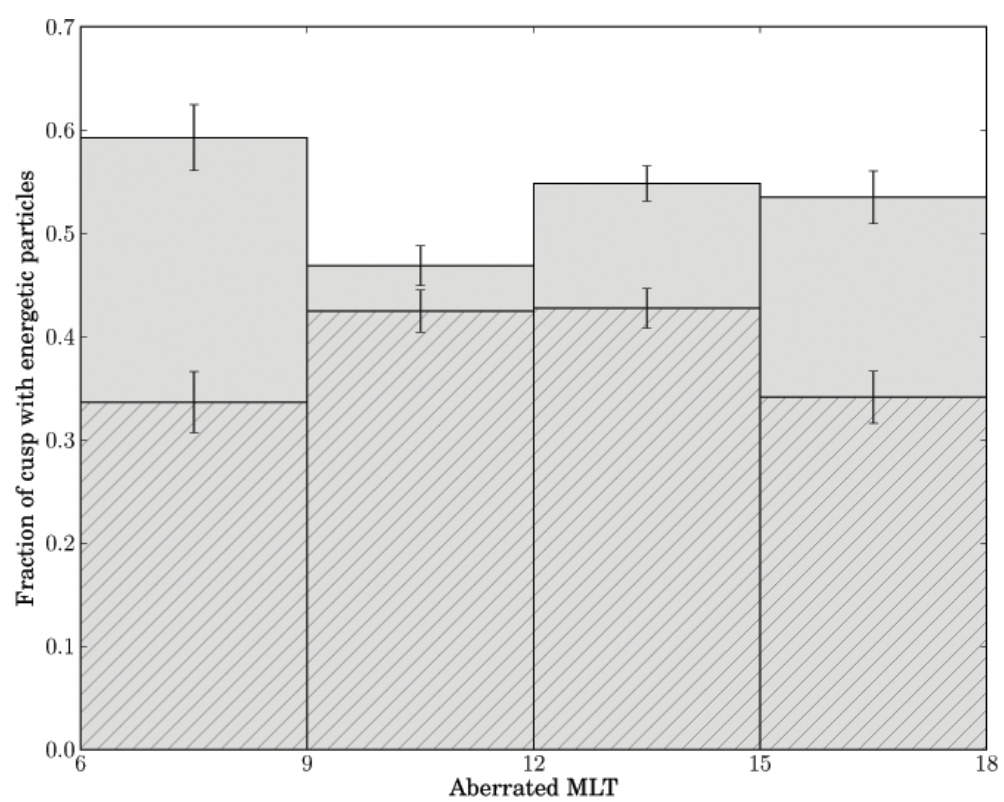

Fig. 6. Fraction of time spent in the cusp which also had energetic particles present, by magnetic local time (corrected for solar wind aberration). Hashed bars had no GOES dipolarizations within preceding four hours; solid bars had at least one dipolarization within the preceding four hours. Error bars are derived from binomial statistics, as explained in the text.

westward motion, so the Polar satellite may have observed them at the dawn side of the cusp first, depending on the details of the drift path bringing them to the cusp.

\section{Conclusions}

- The Polar satellite data in this study support no association between CEP observations and geomagnetic storms. Major CEP contributions to the ring current seem unlikely in light of this result.

- Substorms, as measured by dipolarizations at geosynchronous, are associated at $95 \%$ confidence with CEP observations about three hours post-substorm. Postsubstorms, CEPs are not observed preferentially in dusk sector, as would be expected for ion drift from the tail. Thus energetic ions accelerated in substorms may provide some contribution to CEP populations, but are unlikely to be the dominant source.

- In contrast, substorms determined from auroral signatures show no association with CEPs, possibly from inclusion of substorms or substorm-like events without substantial energetic particle injections.

- There is no suggestion that CEPs contribute to substorm triggering.
Acknowledgements. We are grateful to T. Fritz for useful conversations and providing data from CAMMICE MICS. We thank the POLAR MFE and POLAR HYDRA teams for the use of data from their instruments, the OMNI team for their assimilative solar wind datasets, and J. Gannon for USGS Dst data. We also thank contributors to the NumPy, SciPy, and matplotlib tools, and our SpacePy coauthors at LANL. While at Boston University, JTN was supported by NASA grant NNG05GD23G. We thank the reviewers for detailed, useful comments and suggestions.

Topical Editor R. Nakamura thanks two anonymous referees for their help in evaluating this paper.

\section{References}

Asikainen, T. and Mursula, K.: Energetic particle fluxes in the exterior cusp and the high-latitude dayside magnetosphere: statistical results from the Cluster/RAPID instrument, Ann. Geophys., 23, 2217-2230, doi:10.5194/angeo-23-2217-2005, 2005.

Birn, J., Thomsen, M. F., Borovsky, J. E., Reeves, G. D., McComas, D. J., Belian, R. D., and Hesse, M.: Substorm ion injections: Geosynchronous observations and test particle orbits in three-dimensional dynamic MHD fields, J. Geophys. Res., 102, 2325-2342, doi:10.1029/96JA03032, 1997.

Birn, J., Artemyev, A. V., Baker, D. N., Echim, M., Hoshino, M., and Zelenyi, L. M.: Particle Acceleration in the Magnetotail and Aurora, Space Sci. Rev., 173, p. 20, doi:10.1007/s11214-0129874-4, 2012.

Blake, J. B.: Comment on the paper "Cusp: a new acceleration region of the magnetosphere" by Jiasheng Chen et al., Czech. J. Phys., 49, 675-677, 1999.

Boakes, P. D., Milan, S. E., Abel, G. A., Freeman, M. P., Chisham, G., and Hubert, B.: A statistical study of the open magnetic flux 
content of the magnetosphere at the time of substorm onset, Geophys. Res. Lett., 36, L04105, doi:10.1029/2008GL037059, 2009.

Boakes, P. D., Milan, S. E., Abel, G. A., Freeman, M. P., Chisham, G., and Hubert, B.: A superposed epoch investigation of the relation between magnetospheric solar wind driving and substorm dynamics with geosynchronous particle injection signatures, J. Geophys. Res.-Space Physics, 116, A01214, doi:10.1029/2010JA016007, 2011

Borovsky, J. E., Nemzek, R. J., and Belian, R. D.: The occurrence rate of magnetospheric-substorm onsets - Random and periodic substorms, J. Geophys. Res., 98, 3807-3813, doi:10.1029/92JA02556, 1993.

Brillinger, D. R.: Measuring the association of point processes: a case history, Am. Math. Mon., 83, 16-22, 1976.

Cai, X., Henderson, M. G., and Clauer, C. R.: A statistical study of magnetic dipolarization for sawtooth events and isolated substorms at geosynchronous orbit with GOES data, Ann. Geophys., 24, 3481-3490, doi:10.5194/angeo-24-3481-2006, 2006.

Chang, S., Scudder, J. D., Fuselier, S. A., Fennell, J. F., Trattner, K. J., Pickett, J. S., Spence, H. E., Menietti, J. D., Peterson, W. K., Lepping, R. P., and Friedel, R.: Cusp energetic ions: A bow shock source, Geophys. Res. Lett., 25, 3729-3732, doi:10.1029/98GL52808, 1998.

Chang, S., Scudder, J. D., Fennell, J. F., Friedl, R., Lepping, R. P., Russell, C. T., Trattner, K. J., Fuselier, S. A., Peterson, W. K., and Spence, H. E.: Energetic magnetosheath ions connected to the Earth's bow shock: Possible source of cusp energetic ions, J. Geophys. Res., 105, 5471-5488, doi:10.1029/1999JA900468, 2000.

Chen, J. and Fritz, T. A.: Correlation of cusp MeV helium with turbulent ULF power spectra and its implications, Geophys. Res. Lett., 25, 4113-4116, doi:10.1029/1998GL900122, 1998.

Chen, J. and Fritz, T. A.: High-altitude cusp: The extremely dynamic region in geospace, Surv. Geophys., 26, 71-93, doi:10.1007/s10712-005-1873-5, 2005.

Chen, J., Fritz, T. A., Sheldon, R. B., Spence, H. E., Spjeldvik, W. N., Fennell, J. F., and Livi, S.: A new, temporarily confined population in the polar cap during the August 27, 1996 geomagnetic field distortion period, Geophys. Res. Lett., 24, 1447-1450, doi:10.1029/97GL01369, 1997.

Chen, J., Fritz, T. A., Sheldon, R. B., Spence, H. E., Spjeldvik, W. N., Fennell, J. F., Livi, S., Russell, C. T., Pickett, J. S., and Gurnett, D. A.: Cusp energetic particle events: Implications for a major acceleration region of the magnetosphere, J. Geophys. Res., 103, 69-78, doi:10.1029/97JA02246, 1998.

Cox, D. R.: Some statistical methods connected with series of events, J. Roy. Stat. Soc. B, 17, 129-164, 1955.

Delcourt, D. C. and Sauvaud, J.: Populating of the cusp and boundary layers by energetic (hundreds of $\mathrm{keV}$ ) equatorial particles, $\mathrm{J}$. Geophys. Res., 104, 22635-22648, doi:10.1029/1999JA900251, 1999.

Dmitriev, A. V., Chao, J. K., and Wu, D. J.: Comparative study of bow shock models using Wind and Geotail observations, J. Geophys. Res.Space, 108, 1464, doi:10.1029/2003JA010027, 2003.

Efron, B.: Bootstrap methods: another look at the jackknife, Ann. Stat., 7, 1-26, 1979.

Freeman, M. P. and Morley, S. K.: A minimal substorm model that explains the observed statistical distribution of times between substorms, Geophys. Res. Lett., 31, L12807,
doi:10.1029/2004GL019989, 2004

Frey, H. U., Mende, S. B., Angelopoulos, V., and Donovan, E. F.: Substorm onset observations by IMAGE-FUV, J. Geophys. Res.Space, 109, A10304, doi:10.1029/2004JA010607, 2004.

Fritz, T. A., Chen, J., and Sheldon, R. B.: The Role of the Cusp as A Source for Magnetospheric Particles: A New Paradigm?, Adv. Space Res., 25, 1445-1457, doi:10.1016/S0273-1177(99)00656$0,2000$.

Fritz, T. A., Chen, J., and Siscoe, G. L.: Energetic ions, large diamagnetic cavities, and Chapman-Ferraro cusp, J. Geophys. Res.Space, 108, 1028, doi:10.1029/2002JA009476, 2003.

Fritz, T. A., Fennell, J. F., Livi, S., Roeder, J. L., Daglis, A., Sommer, H., Wilken, B., Kellett, B., Henderson, M., Grande, M., Blake, J. B., Koga, R., and Chen, J.: The Polar CAMMICE Investigation, unpublished manuscript, http://www.bu.edu/buspace/ papers/cammice_instrument.html, 2004.

Gannon, J. L. and Love, J. J.: USGS 1-min Dst index, J. Atmos. Sol.-Terr. Phy., 73, 323-334, doi:10.1016/j.jastp.2010.02.013, 2011.

Hess, W. N.: The radiation belt and magnetosphere, Blaisdell, Waltham, MA, 1968.

Hsu, T. and McPherron, R. L.: An evaluation of the statistical significance of the association between northward turnings of the interplanetary magnetic field and substorm expansion onsets, J. Geophys. Res.-Space, 107, 1398, doi:10.1029/2000JA000125, 2002.

Liou, K.: Polar Ultraviolet Imager observation of auroral breakup, J. Geophys. Res.-Space, 115, A12219, doi:10.1029/2010JA015578, 2010.

Liou, K., Meng, C., Lui, A. T. Y., Newell, P. T., and Wing, S.: Magnetic dipolarization with substorm expansion onset, J. Geophys. Res.-Space, 107, 1131, doi:10.1029/2001JA000179, 2002.

Loewe, C. A. and Prölss, G. W.: Classification and mean behavior of magnetic storms, J. Geophys. Res., 102, 14209-14213, doi:10.1029/96JA04020, 1997.

Morley, S. K. and Freeman, M. P.: On the association between northward turnings of the interplanetary magnetic field and substorm onsets, Geophys. Res. Lett., 34, L08104, doi:10.1029/2006GL028891, 2007.

Morley, S. K., Welling, D. T., Koller, J., Larsen, B. A., Henderson, M. G., and Niehof, J. T.: Spacepy - A Python-based library of tools for the space sciences, in: Proceedings of the 9th Python in science conference, edited by: van der Walt, S. and Millman, J., 39-45, Austin, TX, 2010.

Niehof, J. T. and Morley, S. K.: Determining the significance of associations between two series of discrete events: bootstrap methods, Tech. Rep. LA-14453, Los Alamos National Laboratory, Los Alamos, NM, doi:10.2172/1035497, 2012.

Niehof, J. T., Fritz, T. A., Friedel, R. H. W., and Chen, J.: Size and location of cusp diamagnetic cavities observed by Polar, J. Geophys. Res.-Space, 115, A07201, doi:10.1029/2009JA014827, 2010.

Nykyri, K., Otto, A., Adamson, E., Kronberg, E., and Daly, P.: On the origin of high-energy particles in the cusp diamagnetic cavity, J. Atmos. Sol.-Terr. Phys., 87-88, 70-81, doi:10.1016/j.jastp.2011.08.012, 2012.

O'Brien, T. P. and McPherron, R. L.: An empirical phase space analysis of ring current dynamics: Solar wind control of injection and decay, J. Geophys. Res., 105, 7707-7720, doi:10.1029/1998JA000437, 2000. 
Otto, A., Adamson, E., and Nykyri, K.: Particle Acceleration in Cusp-like Magnetic Trapping Regions, EOS Trans. AGU, 89, Jt. Assem. Suppl., Abstract SM54A-06, 2008.

Papitashvili, N. E. and King, J. H.: A Draft High Resolution OMNI Data Set, EOS Trans. AGU, 87, Jt. Assem. Suppl., Abstract SM33A-02, 2006.

Reeves, G. D., Belian, R. D., and Fritz, T. A.: Numerical tracing of energetic particle drifts in a model magnetosphere, J. Geophys. Res., 96, 13997-14008, doi:10.1029/91JA01161, 1991.

Russell, C. T. (Ed.): The Global Geospace Mission, Kluwer, Dordrecht, 1995.

Scudder, J., Hunsacker, F., Miller, G., Lobell, J., Zawistowski, T., Ogilvie, K., Keller, J., Chornay, D., Herrero, F., Fitzenreiter, R., Fairfield, D., Needell, J., Bodet, D., Googins, J., Kletzing, C., Torbert, R., Vandiver, J., Bentley, R., Fillius, W., McIlwain, C., Whipple, E., and Korth, A.: Hydra - A 3dimensional electron and ion hot plasma instrument for the POLAR spacecraft of the GGS mission, Space Sci. Rev., 71, 459495, doi:10.1007/BF00751338, 1995.

Sergeev, V. A., Pellinen, R. J., and Pulkkinen, T. I.: Steady Magnetospheric Convection: A Review of Recent Results, Space Sci. Rev., 75, 551-604, doi:10.1007/BF00833344, 1996.

Trattner, K. J., Fuselier, S. A., Peterson, W. K., Chang, S., Friedel, R., and Aellig, M. R.: Origins of energetic ions in the cusp, J. Geophys. Res., 106, 5967-5976, doi:10.1029/2000JA003005, 2001.
Trattner, K. J., Petrinec, S. M., Fuselier, S. A., Peterson, W. K., and Friedel, R.: Cusp energetic ions as tracers for particle transport into the magnetosphere, J. Geophys. Res.-Space, 115, A04219, doi:10.1029/2009JA014919, 2010.

Walsh, B. M. and Fritz, T. A.: Cluster energetic electron survey of the high-altitude cusp and adjacent regions, J. Geophys. Res.Space, 116, A12212, doi:10.1029/2011JA016828, 2011.

Whitaker, K. E., Fritz, T. A., Chen, J., and Klida, M.: Energetic particle sounding of the magnetospheric cusp with ISEE1, Ann. Geophys., 25, 1175-1182, doi:10.5194/angeo-25-11752007, 2007.

Zhang, H., Fritz, T. A., Zong, Q.-G., and Daly, P. W.: Stagnant exterior cusp region as viewed by energetic electrons and ions: A statistical study using Cluster Research with Adaptive Particle Imaging Detectors (RAPID) data, J. Geophys. Res.-Space, 110, A05211, doi:10.1029/2004JA010562, 2005.

Zong, Q.-G., Fritz, T. A., Zhang, H., Korth, A., Daly, P. W., Dunlop, M. W., Glassmeier, K.-H., Reme, H., and Balogh, A.: Triple cusps observed by Cluster-Temporal or spatial effect?, Geophys. Res. Lett., 31, L09810, doi:10.1029/2003GL019128, 2004.

Zong, Q. G., Fritz, T. A., Korth, A., Daly, P. W., Dunlop, M., Balogh, A., Fennell, J. F., Sullivan, J. D., Friedel, R. W. H., and Reme, H.: Energetic Electrons as a Field Line Topology Tracer in the High Latitude Boundary/CUSP Region: Cluster Rapid Observations, Surv. Geophys., 26, 215-240, doi:10.1007/s10712-0051879-z, 2005. 